\title{
Scimitar Syndrome: Radiological Diagnosis
}

\author{
M EL GHAIDI*, H EL HAMMAOUI, H.Jalal
}

Radiology department, Mother and child hospital, CHU Med VI, Marrakech

DOI: $10.36347 /$ sasjm.2020.v06i02.008

| Received: 03.02.2020 | Accepted: 10.02.2020 | Published: 22.02 .2020

*Corresponding author: M EL GHAIDI

\section{Abstract}

Scimitar syndrome is characterized by a combination of cardiopulmonary anomalies including partial anomalous pulmonary venous return connection of the right lung to the inferior caval vein leading to the creation of a left-to-right shunt CT. Chest angiography is the reference examination to confirm the diagnosis. We present the radiological aspects of this malformation through these three observations while specifying the interest of the CT angiography in this diagnosis.

Keywords: Scimitar syndrome, CT angigraphy.

Copyright @ 2020: This is an open-access article distributed under the terms of the Creative Commons Attribution license which permits unrestricted use, distribution, and reproduction in any medium for non-commercial use (NonCommercial, or CC-BY-NC) provided the original author and source are credited.

\section{INTRODUCTION}

Scimitar syndrome or Halasz syndrome is a rare disease characterized by partial or total abnormal right pulmonary venous return. The right superior pulmonary vein or the two right veins then drain into the superior vena cava, into the azygos vein, into the inferior vena cava or more rarely directly into the right atrium

\section{OBSERVATION}

This is a 3-month-old male infant who consulted for respiratory distress. At the clinical examination: the infant has a polypnea, tachycardia with a gallop at auscultation, without weight loss.
The chest x-ray showed cardiomegaly. The echo-heart showed cardiomegaly associated with CIA and PAH. Chest CT angiography: the two right pulmonary veins discharge into the inferior vena cava, associated with dextrocardia, cardiomegaly, moderate hypoplasia of the right lung, and right intra-lobar pulmonary sequestration (figure 1).

The angiography confirmed the diagnosis of scimitar syndrome with embolization of the pulmonary sequestrum.

The evolution was marked by the disappearance of PAH with good clinical evolution. The patient was put under surveillance.

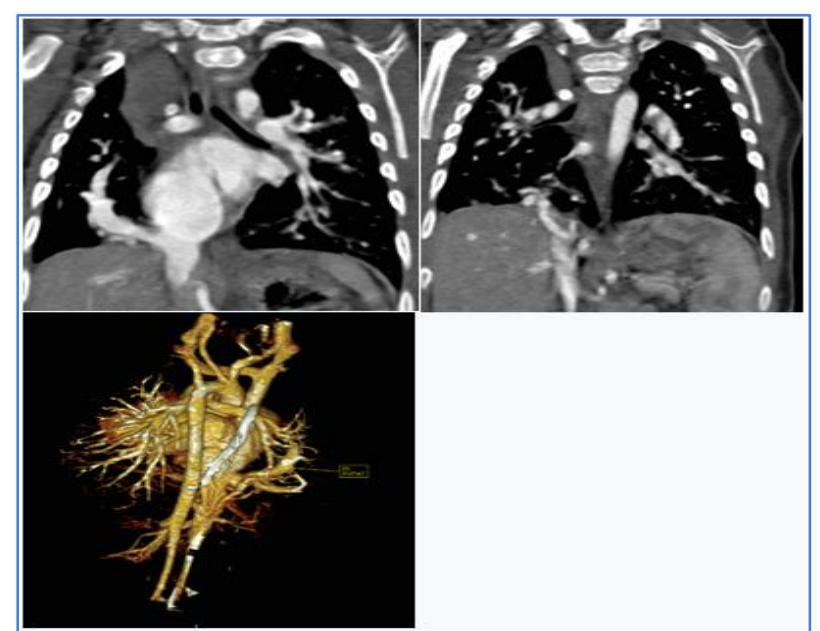

Fig-1, 2, 3: CT scan in coronal reconstruction and 3D, after injection of the contrast product: Two straight pulmonary veins enter the IVC associated with pulmonary sequestration (Figure 2) 


\section{Observation 2}

This is a 2-year-old female child who has been hospitalized in intensive care for acute respiratory distress secondary to foreign body inhalation. Chest Xray showed cardiomegaly with opacities of right lung. Chest CT angiography showed: the two right pulmonary veins discharge into the inferior vena cava associated with dextrocardia, CIA, hypoplasia of the right lung with intra-lobar pulmonary sequestration. The patient was referred to the cardio vascular surgery department for additional care.

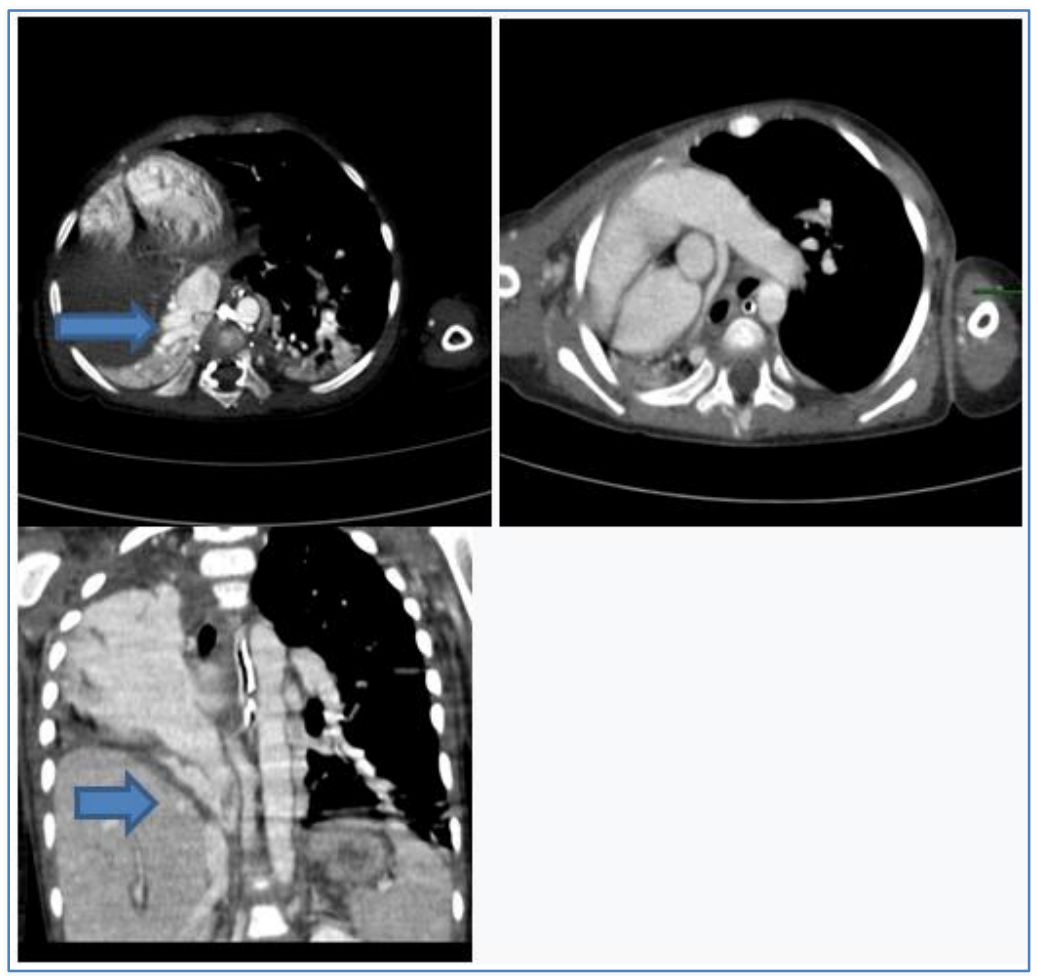

Fig-4, 5, 6: CT scan in coronal reconstruction, after injection of the contrast product: Two straight pulmonary veins enté the IVC associa ted with hypoplasic right pulmonary artery (Figure 2).

\section{Observation 3}

18-month-old infant who consulted for recurrent respiratory infections. On standard radiography: Opaque right lung. The $\mathrm{CT}$ angioscanner objected a right partial syndrome scimitar: the two right pulmonary veins flow into the inferior vena cava associated with dextrocardia, CIA, right lung hypoplasia, and intra-lobar pulmonary sequestration.

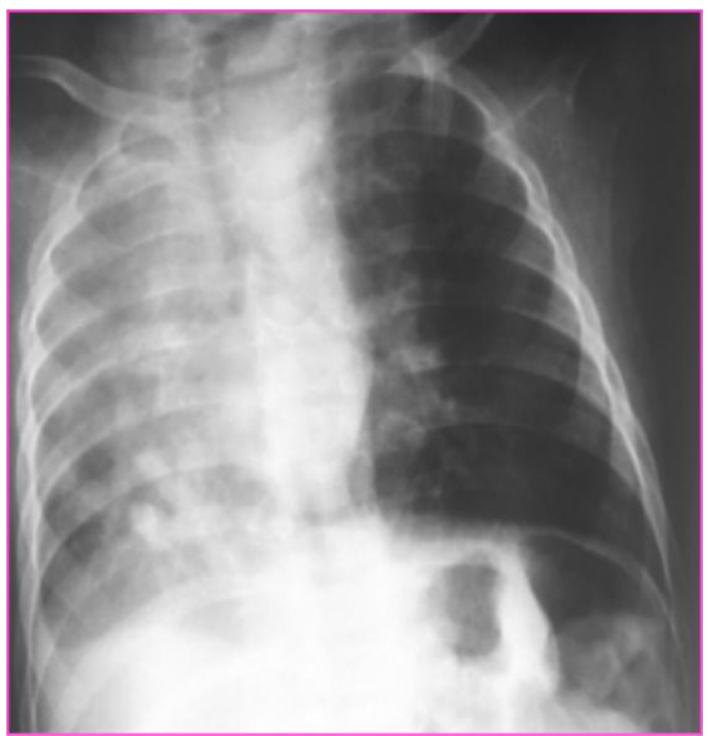

Fig-4: Chest $X$ ray: opacities of the right hemifield, deviated aspect of the trachea and mediastinum 

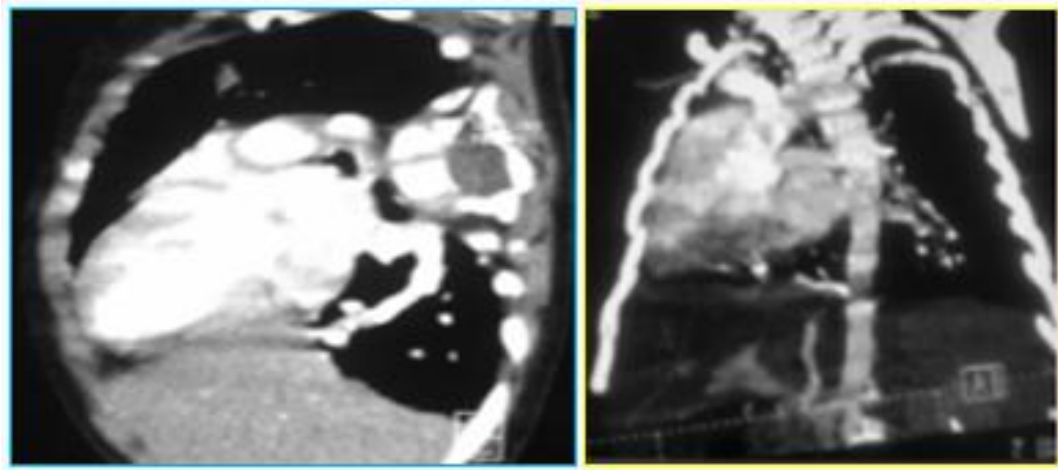

Fig-7,8: One right pulmonary vein flows into the right atrium

\section{DISCUSSION}

Scimitar syndrome is a complex group of veno-pulmonary abnormalities also grouped together as Felson vein-lobar syndrome or Halasz syndrome known for more than a century [1].

This syndrome corresponds above all to an abnormal right pulmonary venous return (RVPA) which classically associates itself with other malformations such as: a cardiac dextro-position by rotation, a straight lung hypoplasia, a bronchopulmonary sequestration and/or cardiac malformations, including inter-ear communication (AIC) [1].

The incidence is probably 1 to 3 per 100,000 live. The association of a scimitar-type RVPA with sequestration is described in $50 \%$ of patients with this syndrome [3]. The diagnosis is evoked on the chest Xray in front of the presence of a right basal arciform opacity, going from the hilar region to the diaphragmatic dome giving a Turkish sword-like appearance[1].

The clinical expression is very variable, ranging from an intolerance from the first days of life to a chance discovery in adults; it depends on the importance of pulmonary hypoplasia and arteriovenous fistula through sequestration [3].

The diagnosis is evoked on X-ray chest of the presence of a right basal arciform opacity, going from the hilar region to the diaphragmatic dome [4].

Chest angiography is the reference examination to confirm the diagnosis, it allows morphological analysis of the pulmonary parenchyma, bronchial tree, pulmonary and systemic vascular anatomy [4, 5]. cardiac MRI also provides excellent visualization of the vascular anatomy of this vascular malformation complex [5] the close similarity with conventional angiography suggests that ccmagnetic resonance angiography can be used as a non-invasive diagnostic tool to confirm the diagnosis of this vascular malformation complex [5].

The curative treatment of scimitar syndrome involves in some cases heavy surgery involving a reimplantation of the pulmonary vein in the left atrium and surgery of other possible cardiovascular malformations associated including surgery of pulmonary sequestration and closure of CIA[6].

\section{REFERENCES}

1. Holt PD, Berdon WE, Marans Z, Griffiths S, Hsu D. Scimitar vein draining to the left atrium and a historical review of the scimitar syndrome. Pediatr Radiol. 2004;34:409-13.

2. Dupuis C, Charaf LA, Breviere GM, Abou P, Remy-Jardin M, Helmius G. The 'adult' form of the scimitar syndrome. Am J Cardiol. 1992 Aug;70(4):502-7.

3. Deutscher C, Helms P, Rebeuh J, Livolsi A, Donato L, Becmeur F, Fischbach M. Le syndrome du Cimeterre: diagnostic différentiel d'une bronchiolite. Archives de pédiatrie. 2008 June; 15 (5): 983-84.

4. Syndrome de cimeterre: à propos d'un cas et revue de la littérature. Ibtihale Benjouad, Ikram Taam, Khaoula El Ataouna, Mohamed Mahi, Touriya Amil, Rachida Saouab The Pan African Medical Journal. 2016

5. Sinha R, Singh P, Bhatnagar AK, Batra A. Scimitar syndrome: Imaging by magnetic resonance angiography and Doppler echocardiography. Indian J Dis Allied Sci. 2004;46:283-6.

6. Dupuis C, Charaf LA, Breviere GM, Abou P, Remy-Jardin M, Helmius G. The 'adult' form of the scimitar syndrome. Am J Cardiol. 1992 Aug;70(4):502-7. 